\title{
Prevalence of Oxaliplatin-induced Chronic Neuropathy and Influencing Factors in Patients with Colorectal Cancer in Iran
}

\author{
Ali Shahriari-Ahmadi ${ }^{1}$, Ali Fahimi ${ }^{1}$, Mehrdad Payandeh ${ }^{2}$, Masoud Sadeghi ${ }^{3 *}$
}

\begin{abstract}
Background: The chemotherapeutic agent oxaliplatin can cause acute and chronic forms of peripheral neuropathy. The aim of this study was to evaluate the incidence of chronic neuropathy and its risk factors in colorectal cancer (CRC) patients treated with FOLFOX or XELOX regimens in the Oncology Ward of Hazrate-Rasoul Hospital in Tehran. Materials and Methods: A total of 130 patients with CRC were entered into our study, aged over 18 years, without history of receiving other neurotoxic agents or other predisposing factors such as diabetes or neurologic diseases and kidney and liver dysfunction. For the FOLFOX regimen, patients received oxaliplatin, $85 \mathrm{mg} / \mathrm{m} 2$, every 2 weeks for 12 courses and with the XELOX regimen, oxaliplatin was $130 \mathrm{mg} / \mathrm{m}^{2}$, every 3 weeks for 8 courses. Based on Common Toxicity Criteria (CTC or NCI-CTC v.3), the patients were divided into 5 groups (grades) based on the severity of their symptoms. Results: Fifty-seven patients (43.8\%) were male and 73(56.2\%) female. Some 19 patients $(14.7 \%)$ had $B M I<20,97(74.6 \%)$ were between $20-25$ and $14(10.8 \%) \geq 25$. In 105 patients $(80.7 \%)$ neuropathy was found. There was significant correlation between BMI, hypomagnesaemia and especially, severity of anemia in patients with neuropathy compared to those without. Conclusions: Oxaliplatin regimens can induce chronic neuropathy in CRC patients, with anemia, high BMI and hypomagnesaemia as risk factors that can predispose to this kind of neurotoxicity.
\end{abstract}

Keywords: Anemia - chronic neuropathy - colorectal cancer - oxaliplatin

Asian Pac J Cancer Prev, 16 (17), 7603-7606

\section{Introduction}

Colorectal cancer (CRC) is one of the most common cancers and is the 2nd leading cause of cancer death in men and women in the United States (Stone et al., 2004) and also is third common cancer in women and 5th in Iranian men (Azadeh et al., 2008).

Although all platinum analogs are potentially neurotoxic, oxaliplatin is associated with a unique spectrum of neurologic symptoms (Park et al., 2009). The peripheral nervous system can be vulnerable to the toxic action of several drugs since it is not protected as effectively as the central nervous system from noxious exogenous agents. Drug-induced neurotoxicity can affect the nerve fibers or the neuronal bodies (generally the dorsal root ganglia of the primary sensory neurons). Among the neurotoxic drugs antineoplastic agents represent a major clinical problem, given their widespread use and the potential severity of their toxicity (Argyriou et al., 2012). Infusion of the chemotherapeutic agent oxaliplatin leads to an acute and a chronic form of peripheral neuropathy. Acute oxaliplatin neuropathy is characterized by sensory paresthesias and muscle cramps that are notably exacerbated by cooling. Painful dysesthesias are rarely reported for acute oxaliplatin neuropathy, whereas a common symptom of chronic oxaliplatin neuropathy is pain (Sittl et al., 2012). Calcium and magnesium solutions are an effective and convenient means of treating and reducing the severity of neuropathic symptoms( Cersosimo, 2005).

Capecitabin is an oral fluoropyrimidine with similar efficacy to bolus 5-fluorouracil/folinic acid (5-FU/FA) in the first-line treatment of metastatic colorectal cancer and as adjuvant therapy for stage III colon cancer and also the efficacy of capecitabine and a 3-weekly dose of oxaliplatin (XELOX regimen) is also non-inferior to 5-FU/ FA plus oxaliplatin (FOLFOX) in the first- and secondline treatment of patients with metastatic colorectal cancer (Cassidy et al., 2011).

The aim of study is to evaluated incidence of chronic neuropathy and its risk factors in CRC patients that treated with FOLFOX or XELOX regimen in Tehran province, Iran.

\section{Materials and Methods}

Of all referred CRC patients to Oncology Clinic, Hazrat-e-Rasoul Hospital, Tehran, Iran, 130 CRC patients were entered to our study that were treating with 


\section{Ali Shahriari-Ahmadi et al}

Oxaliplatin, age $>18$ years, not having of history of other chemotherapy and also these patients didn't have previous neuropathy history, polymyositis, kidney and liver failure. The patients were treated with adjuvant chemotherapy regimen of FOLFOX (5-Flourouracil, leucovorin, oxaliplatin) or XELOX (capecitabine plus oxaliplatin). In FOLFOX regimen patients received oxaliplatin, $85 \mathrm{mg} /$ $\mathrm{m} 2$ every 2 weeks for 12 courses and in XELOX regimen, $130 \mathrm{mg} / \mathrm{m} 2$ every 3 weeks for 8 courses. The evaluation for neuropathy was done before starting treatment, after fourth course of XELOX or sixth course of FOLFOX, at the end of treatment; and also 3 months and 6 month after last course. Before treatment, complete blood count (CBC), serum calcium and serum magnesium levels were evaluated in all of the patients.

Based on Common Toxicity Criteria (CTC or NCICTC v.3), the patients were divided to 5 groups based on the grade of neurotoxicity (Table 1)(Vincenzi et al., 2013). Also, parameters definition(hypocalcaemia, hypomagnesaemia, anemia, diabetes, alcohol consumption) has been written in Table 2 (Vincenzi et al., 2013). In this study, we evaluated only chronic peripheral neuropathy by oxaliplatin.

The correlation between variables was done by IBM SPSS statistics v.19 (Chi-square test) that $\mathrm{P}<0.05$ was statistically significant.

\section{Results}

Of 130 CRC patients, 67 patients $(51.5 \%)$ had age $>60$ years and 63 patients $(48.5 \%)$ had $\geq 60$ years. Fifty-seven patients $(43.8 \%)$ were male and 73(56.2\%) female. Body mass index (BMI) was divided to 3 groups that 19 patients $(14.65 \%)<20,97(74.6 \%)$ between $20-25$ and $14(10.8 \%)$ $\geq 25$. In our patients, 105 patients $(80.7 \%)$ had neuropathy, 28 patients $(21.5 \%)$ had diabetes, 98 patients $(75.3 \%)$ had anemia, 25 patients(19.3) had hypocalcaemia, 18 patients $(13.8 \%)$ had hypomagnesaemia and 5 patients $(3.8 \%)$ were consuming alcohol (Table 1).

Table 1. The Characteristics of the Patients $(n=130)$

\begin{tabular}{lrr}
\hline Variables & & $\mathrm{n}(\%)$ \\
\hline Age & $>60$ & $67(51.5)$ \\
Sex & $\leq 60$ & $63(48.5)$ \\
& Male & $57(43.8)$ \\
BMI* & Female & $73(56.2)$ \\
& $<20$ & $19(14.6)$ \\
Neuropathy & $20-25$ & $97(74.6)$ \\
& $\geq 25$ & $14(10.8)$ \\
Diabetes & Yes & $105(80.7)$ \\
& No & $25(19.3)$ \\
Anemia & Yes & $28(21.5)$ \\
& No & $102(78.5)$ \\
Hypocalcaemia & Yes & $98(75.3)$ \\
& No & $32(24.7)$ \\
Hypomagnesaemia & Yes & $25(19.3)$ \\
& No & $105(80.7)$ \\
Alcohol consumption & Yes & $18(13.8)$ \\
& No & $112(86.2)$ \\
& Yes & $5(3.8)$ \\
\hline
\end{tabular}

Table 2. The Correlation between Neuropathy and Some other Variables in Colorectal Cancer Patients $(\mathbf{n}=130)$

\begin{tabular}{lccc}
\hline Variables, $\mathrm{n}(\%)$ & \multicolumn{2}{c}{ Neuropathy } & P-value* \\
& Yes(n=105) & No(n=25) & \\
\hline Age & & & 0.68 \\
$\quad>60$ & $52(49.5)$ & $15(60)$ & \\
$\quad \leq 60$ & $53(50.5)$ & $10(40)$ & \\
Sex & & & 0.83 \\
$\quad$ Male & $52(49.5)$ & $5(20)$ & \\
Female & $53(50.5)$ & $20(80)$ & \\
BMI* & & & 0.003 \\
$\quad<20$ & $8(7.6)$ & $11(44)$ & \\
$20-25$ & $84(80)$ & $13(52)$ & \\
$\quad \geq 25$ & $13(12.4)$ & $1(4)$ & \\
Diabetes & & & 0.54 \\
$\quad$ Yes & $22(21)$ & $6(24)$ & \\
$\quad$ No & $83(79)$ & $19(76)$ & \\
Anemia & & & 0.004 \\
$\quad$ Yes & $85(81)$ & $13(52)$ & \\
$\quad$ No & $20(19)$ & $12(48)$ & \\
Hypocalcaemia & & & 0.148 \\
$\quad$ Yes & $20(19)$ & $5(20)$ & \\
$\quad$ No & $85(81)$ & $20(80)$ & \\
Hypomagnesaemia & & & 0.045 \\
Yes & $17(16.2)$ & $1(4)$ & \\
$\quad$ No & $88(83.8)$ & $24(96)$ & 0.82 \\
Alcohol consumption & & & \\
$\quad$ Yes & $2(1.9)$ & $3(12)$ & \\
$\quad$ No & $103(98.1)$ & $22(88)$ & \\
\hline
\end{tabular}

*Chi-square test

Table 3. The Prevalence of Grades of Neuropathy with a Number of Variables in Colorectal Cancer Patients

\begin{tabular}{|c|c|c|c|c|}
\hline Variables, $\mathrm{n}(\%)$ & Grade 1 & Grade 2 & Grade 3 & P-value* \\
\hline Age & & & & 0.78 \\
\hline$>60$ & $8(47.1)$ & $27(47.4)$ & $17(54.8)$ & \\
\hline$\leq 60$ & $9(52.9)$ & $31(52.6)$ & $13(45.2)$ & \\
\hline Sex & & & & 0.882 \\
\hline Male & $8(47.1)$ & $28(48.3)$ & $16(53.3)$ & \\
\hline Female & $9(52.9)$ & $30(51.7)$ & $14(46.7)$ & \\
\hline Anemia & & & & 0 \\
\hline Yes & $8(47.1)$ & $52(89.7)$ & $25(83.3)$ & \\
\hline No & $9(52.9)$ & $6(10.3)$ & $5(16.7)$ & \\
\hline \multicolumn{4}{|c|}{ Hypomagnesaemia } & 0.42 \\
\hline Yes & $4(23.5)$ & $7(12.1)$ & $6(20)$ & \\
\hline No & $13(76.5)$ & $51(87.9)$ & $24(80)$ & \\
\hline \multicolumn{4}{|l|}{ Hypocalcaemia } & 0.267 \\
\hline Yes & $4(30)$ & $8(13.8)$ & $8(23.5)$ & \\
\hline No & $9(69.2)$ & $50(86.2)$ & $26(76.5)$ & \\
\hline \multicolumn{4}{|l|}{ Diabetes } & 0.072 \\
\hline Yes & $7(41.2)$ & $9(15.5)$ & $6(20)$ & \\
\hline No & $10(58.8)$ & $49(84.5)$ & $24(80)$ & \\
\hline
\end{tabular}

Table 4. The Correlation between Neuropathy with Chemotherapy Regimens

\begin{tabular}{lc}
\hline Chemotherapy regimen, $\mathrm{n}(\%)$ & Total \\
\hline FOLFOX & $87(66.9)$ \\
XELOX & $43(33.1)$ \\
\hline Total & $130(100)$ \\
\hline
\end{tabular}


The correlation between neuropathy with a number of variables in CRC has been shown in Table 2. In the patients with neuropathy, more had high BMI that this correlation was statistically significant $(\mathrm{P}=0.003)$. In addition, anemia and hypomagnesaemia in the patients with neuropathy were more than the patients without neuropathy $(\mathrm{P}<0.05)$.

Table 3 shows the distribution of grades of neuropathy in relation with other of variables in CRC patients. In our study, there were no grade 0 and grade 4 . There was the close correlation between anemia and severity of neuropathy $(\mathrm{P}=0.000)$.

Of 133 CRC patients, $87(66.9 \%)$ patients were treated with FOLFOX regimen and 43(33.1\%) patients with XELOX regimen (Table 4). There was no correlation between regimens with neuropathy $(\mathrm{P}=0.14)$.

\section{Discussion}

CRC is the third most common neoplastic disease worldwide. It is one of the leading causes of cancer mortality, accounting for about $10 \%$ of all cancer deaths, with approximately $40 \%-50 \%$ of all cases diagnosed as metastatic (Payandeh et al., 2015). Peripheral neuropathy ranks among the most common non-haematological adverse effects of a number of effective chemotherapeutic agents, including platinum compounds, taxanes and vinca alkaloids. Chemotherapy-induced peripheral neuropathy (CIPN) may result from a variety of mechanisms and may be related to causal factors, such as single dose per course, cumulative dose and risk factors including treatment schedule, prior or concomitant administration of other neurotoxic agents, age and pre-existing peripheral neuropathy of other causes (Argyriou et al., 2010). The advent of monoclonal antibodies such as cetuximab and bevacizumab has further broadened the treatment horizon for colorectal cancer and they are the focus of the on-going randomized studies in adjuvant therapy of colon cancer (Chau I and Cunningham D.,2006). Adding oxaliplatin to a regimen of fluorouracil and leucovorin improves the adjuvant treatment of colon cancer (André et al., 2004).

In a study, (Rothenberg et al., 2008) 627 patients were randomly assigned to receive XELOX $(\mathrm{n}=313)$ or FOLFOX $(n=314)$ following disease progression/recurrence or intolerance to irinotecan-based chemotherapy. XELOX was non-inferior to FOLFOX when administered as second-line treatment in patients with metastatic colorectal cancer.In other study, (Argyriou et al., 2007) twenty-five adult patients scheduled to be treated with 12 courses of the oxaliplatin-based regimen, FOLFOX-4, for metastatic colon cancer. The results indicate that the majority of patients treated with the FOLFOX-4 regimen would manifest an axonal, predominately sensory peripheral neuropathy, of mild to moderate severity.

420 untreated CRC patients were randomized to receive a 2-hour infusion of leucovorin $\left(200 \mathrm{mg} / \mathrm{m}^{2} / \mathrm{d}\right)$ followed by a 5 -fluorouracil bolus $\left(400 \mathrm{mg} / \mathrm{m}^{2} / \mathrm{d}\right)$ and 22-hour infusion $\left(600 \mathrm{mg} / \mathrm{m}^{2} / \mathrm{d}\right)$ for 2 consecutive days every 2 weeks, either alone or together with oxaliplatin $85 \mathrm{mg} / \mathrm{m}^{2}$ as a 2-hour infusion on day 1 . The grade 3 neurosensory toxicity was $18.2 \%$ versus $0 \%$. Therefore, leucovorin, 5-fluorouracil and oxaliplatin combination seems beneficial as first-line therapy in advanced colorectal cancer (Gramont et al., 2000).

A research showed that oxaliplatin/leucovorin/5fluorouracil regimen demonstrated substantial efficacies and manageable toxicity profiles in the first-line treatment of patients with advanced CRC. (7) Other report showed that there was oxaliplatin-induced peripheral neuropathy in patients with CRC treated with either FOLFOX or XELOX and the incidence of acute neurotoxicity during FOLFOX therapy was similar to XELOX, but FOLFOX was associated with increased incidence of chronic neurotoxicity, compared with XELOX-treated patients (Meyerhardt et al., 2004). Incidence of acute peripheral neuropathy during XELOX appears similar to FOLFOX, but chronic peripheral neuropathy in adjuvant patients may be more common with XELOX (Storey et al., 2010).

100 patients treated with adjuvant FOLFOX and 266 patients treated with XELOX. The median cumulative dose of oxaliplatin was $796 \mathrm{mg} / \mathrm{m}^{2}$ (range, 85 to $1,583 \mathrm{mg}$ / $\mathrm{m}^{2}$ ). Overall, 43 patients discontinued chemotherapy due to toxicity (Baek et al., 2010). Patients with metastatic CRC received first-line XELOX in 3-week treatment cycles: intravenous oxaliplatin $130 \mathrm{mg} / \mathrm{m}^{(2)}$ (day 1) followed by oral capecitabine $1,000 \mathrm{mg} / \mathrm{m}^{(2)}$ twice daily (day 1, evening, up to day 15, morning). XELOX safety was predictable and similar to the FOLFOX4 regimen, except that myelosuppression was uncommon with XELOX (grade 3 or 4 neutropenia, 7\%). Most adverse events were mild to moderate, the most common being acute sensory neuropathy (85\%) (Cassidy et al., 2004). Also, in a study reported that that 5-fluorouracil or its active metabolites were contributing factors to the peripheral neuropathy (Saif et al., 2001). In our study, of 133 CRC patients treated with FOLFOX or XELOX, overall 105 patients (80.7\%) had chronic neuropathy and also there was correlation between these regimens with incidence of neuropathy $(\mathrm{P}>0.05)$. Based on these results, it is clear that oxaliplatin in both regimens can induce neuropathy with high incidence especially chronic neuropathy in CRC patients, but dose and number of courses for oxaliplatin in chemotherapy regimens can change percentage of neuropathy in patients. In future studies can check this subject that a study reported chronic neuropathy is cumulative and is most commonly seen in patients who have received total doses $>$ or $=540 \mathrm{mg} / \mathrm{m}^{2}$ (Cersosimo, 2005).

Anemia, albumin level, chronic renal failure, number of cycles, alcohol consumption and hypomagnesaemia were variables that in CRC patients had significant correlation with grade of neuropathy (high incidence for these variables equals with high grade) (Vincenzi et al., 2013). In CRC patients, greater BMI potentially associate with persistent neuropathy, but in contrast to weight, height was not an informative factor; as a result, BMI (weight adjusted for height) did not predict neuropathy as efficiently as weight alone did (Alejandro et al., 2013). A number studies on the mechanism of peripheral sensory neurotoxicity (PSN) reported that calcium and magnesium replacement effectively reduced chronic PSN, suggesting that these supplements are efficacious (Gamelin et al., 2002; Gamelin et al., 2004; Kono et al., 2010). In our 
study, anemia just had significant correlation with grade of neuropathy. In CRC patients with anemia, there is high risk for incidence of neuropathy with high grade. In addition, BMI and hypomagnesaemia had significant correlation with incidence of neuropathy, but there is correlation these variables with grade.

In conclusion, Oxaliplatin regimens can induce chronic neuropathy in CRC patients, but these are almost similar in incidence of neuropathy. Anemia, high BMI and hypomagnesaemia are risk factors that can predispose to this kind of neurotoxicity. Oncologists should pay special attention to high incidence of neuropathy in CRC patients that have anemia and receive oxaliplatin regimens.

\section{References}

Alejandro LM, Behrendt CE, Chen K, Openshaw H, Shibata S (2013). Predicting acute and persistent neuropathy associated with oxaliplatin. Am J Clin Oncol, 36, 331-7.

André T, Boni C, Mounedji-Boudiaf L, et al (2004). Multicenter International Study of Oxaliplatin/5-Fluorouracil/Leucovorin in the Adjuvant Treatment of Colon Cancer (MOSAIC) Investigators. Oxaliplatin, fluorouracil, and leucovorin as adjuvant treatment for colon cancer. $N$ Engl J Med, 350, 2343-51.

Argyriou AA, Bruna J, Marmiroli P, Cavaletti G(2012). Chemotherapy-induced peripheral neurotoxicity (CIPN): an update. Crit Rev Oncol Hematol, 82, 51-77.

Argyriou AA, Polychronopoulos P, Iconomou G, et al (2007). Incidence and characteristics of peripheral neuropathy during oxaliplatin-based chemotherapy for metastatic colon cancer. Acta Oncol, 46, 1131-7.

Argyriou AA, Zolota V, Kyriakopoulou O, Kalofonos HP(2010). Toxic peripheral neuropathy associated with commonly used chemotherapeutic agents. J BUON, 15, 435-46.

Azadeh S, Moghimi-Dehkordi B, Fatem SR, et al (2008). Colorectal cancer in Iran: an epidemiological study. Asian Pac J Cancer Prev, 9, 123-6.

Baek KK, Lee J, Park SH, et al (2010). Oxaliplatin-induced chronic peripheral neurotoxicity: a prospective analysis in patients with colorectal cancer. Cancer Res Treat, 42, 185-90.

Cassidy J, Clarke S, Díaz-Rubio E, et al (2011). XELOX vs FOLFOX-4 as first-line therapy for metastatic colorectal cancer: NO16966 updated results. Br J Cance, 105, 58-64.

Cassidy J, Tabernero J, Twelves C, et al (2004). XELOX (capecitabine plus oxaliplatin): active first-line therapy for patients with metastatic colorectal cancer. J Clin Oncol, 22, 2084-91.

Cersosimo RJ(2005). Oxaliplatin-associated neuropathy: a review. The Annals of Pharmacology, 39, 128-35.

Chau I, Cunningham D(2006). Adjuvant therapy in colon cancer-what, when and how? Ann Oncol, 17, 1347-59.

de Gramont A, Figer A, Seymour M, et al (2000). Leucovorin and fluorouracil with or without oxaliplatin as first-line treatment in advanced colorectal cancer. J Clin Oncol, 18, 2938-47.

Gamelin L, Boisdron-Celle M, Delva R, et al (2004). Prevention of oxaliplatin-related neurotoxicity by calcium and magnesium infusions: a retrospective study of 161 patients receiving oxaliplatin combined with 5-Fluorouracil and leucovorin for advanced colorectal cancer. Clin Cancer Res, 10, 4055-61.

Gamelin E, Gamelin L, Bossi L, Quasthoff S(2002). Clinical aspects and molecular basis of oxaliplatin neurotoxicity: current management and development of preventive measures. Semin Oncol, 29, 21-33.

Kalofonos HP, Aravantinos G, Kosmidis P, et al (2005).
Irinotecan or oxaliplatin combined with leucovorin and 5-fluorouracil as first-line treatment in advanced colorectal cancer: a multicenter, randomized, phase II study. Ann Oncol, 16, 869-77.

Kono T, Satomi M, Asama T, et al (2010). Cetuximabinduced hypomagnesaemia aggravates peripheral sensory neurotoxicity caused by oxaliplatin. J Gastrointest Oncol, 1,97-101.

Meyerhardt JA, Tepper JE, Niedzwiecki D, et al (2004). Impact of body mass index on outcomes and treatment-related toxicity in patients with stage II and III rectal cancer: findings from Intergroup Trial 0114. J Clin Oncol, 22, 648-57.

Park SB, Goldstein D, Lin CS, et al(2009). Acute abnormalities of sensory nerve function associated with oxaliplatininduced neurotoxicity. J Clin Oncol, 27, 1243-9.

Payandeh M, Sadeghi M, Sadeghi E, Gholami F (2015). Analysis of KRAS, BRAF and NRAS in Patients with Colorectal Cancer: the First Report of Western Iran. Am J Cancer Prev, 3, 19-22.

Rothenberg ML, Cox JV, Butts C, et al (2008). Capecitabine plus oxaliplatin (XELOX) versus 5-fluorouracil/folinic acid plus oxaliplatin (FOLFOX-4) as second-line therapy in metastatic colorectal cancer: a randomized phase III noninferiority study. Ann Oncol, 19, 1720-6.

Saif MW, Wilson RH, Harold N, et al (2001). Peripheral neuropathy associated with weekly oral 5-fluorouracil, leucovorin and eniluracil. Anticancer Drugs, 12, 525-31.

Sittl R, Lampert A, Huth T, et al (2012). Anticancer drug oxaliplatin induces acute cooling-aggravated neuropathy via sodium channel subtype $\mathrm{Na}(\mathrm{V}) 1.6$-resurgent and persistent current. Proc Natl Acad Sci U S A, 109, 6704-9.

Stone WL, Krishnan K, Campbell SE, et al (2004). Tocopherols and the treatment of colon cancer. Ann NY Acad Sci, 1031, 223-33.

Storey DJ, Sakala M, McLean CM, et al (2010). Capecitabine combined with oxaliplatin (CapOx) in clinical practice: how significant is peripheral neuropathy? Ann Oncol, 21, 1657-61.

Vincenzi B, Frezza AM, Schiavon G, et al (2013). Identification of clinical predictive factors of oxaliplatin-induced chronic peripheral neuropathy in colorectal cancer patients treated with adjuvant Folfox IV. Support Care Cancer, 21, 1313-9. 